\title{
Fast Decay of Adatom Islands and Mounds on Cu(111): A New Effective Channel for Interlayer Mass Transport
}

\author{
M. Giesen,* G. Schulze Icking-Konert, and H. Ibach \\ Institut für Grenzflächenforschung und Vakuumphysik, Forschungszentrum Jülich, D-52425 Jülich, Germany
}

(Received 14 August 1997)

\begin{abstract}
We report on the observation of a new and very effective mechanism of interlayer mass transport which bypasses the Schwoebel-Ehrlich barrier for the diffusion of atoms over step edges. The channel for a rapid mass transport opens when a two-dimensional island engaged in a random walk on a surface touches the boundary of a descending step. The decay rate of the island then increases by about 2 orders of magnitude. Even entire mounds can disappear in a very short time due to ledge contact events caused by equilibrium fluctuations of step edges. [S0031-9007(97)05053-9]

PACS numbers: 68.35.Fx, 61.16.Ch, 82.65.Dp
\end{abstract}

The morphology of epitaxially grown thin films on solid surfaces is frequently controlled by kinetics rather than equilibrium thermodynamics. A delicate interplay between nucleation, diffusion, and the mass exchange between terraces of different height can cause surprisingly complex morphological features. The mass exchange between terraces is often hindered by an additional activation barrier for the diffusion of adatoms across step edges [Schwoebel-Ehrlich (SE) barrier [1,2]] which gives rise to a growth in the form of three-dimensional (3D) pyramidal structures ("mounds"). These mounds display a characteristic slope, which either becomes steeper with continuing deposition or remains constant [3-5]. On vicinal surfaces, the SE barrier stabilizes step flow growth with equally spaced steps, but may cause a meandering instability of the steps and the appearance of new facets ("BalesZangwill" instability) [6,7]. A significant SE barrier and 3D growth at lower temperatures has been reported for the (111) surfaces of Pt [8], $\mathrm{Rh}$ [9], $\mathrm{Ag}$ [10], and $\mathrm{Cu}$ [11]. The magnitude of the SE barrier can be determined experimentally by measuring the decay of vacancy islands [12] or by the decay of islands placed in the vicinity of a descending step in relation to the decay of islands next to an ascending step when the experimental data are numerically analyzed using the continuum theory for the diffusion limited decay [13]. On the $\mathrm{Cu}(111)$ surface, e.g., the SE barrier was determined to $0.12 \mathrm{eV}$ [13] for steps with (100) orientation. Theoretical investigations $[14,15]$ as well as earlier experimental results [8] indicate that the SE barrier depends on the type and roughness of the step. For steps on a (111) surface, exchange diffusion is favored over hopping. The highest activation energy is obtained for straight (100) steps and the lowest for kinks in a (111) step [14,15].

Previous experimental and theoretical studies on the evolution of morphological features during growth and decay are based on the concept of an interlayer mass transport with single adatoms on the terraces (or vacancies [16]) as the diffusing species. In this Letter, we report the observation of a new, effective mechanism of interlayer mass transport in the decay of islands and mounds which bypasses the bottleneck of adatom diffusion over the SE barrier: By virtue of the rather rapid mass transport alongside steps [17], adatom islands engage in a random walk across the surface [18,19]. Because of this random walk, every once in a while an island placed on top of another island touches a descending step of the island below. Then, a new channel for interlayer mass transport opens, leading to a dramatic increase in the decay rate and frequently to complete disappearance of the upper island. The new channel for interlayer mass transport also causes the rapid disappearance of entire mounds. In that case, the decay is controlled by the decay of the lowest island of the mound and the slope of the mound remains constant during the decay.

The decay of mounds and islands on the $\mathrm{Cu}(111)$ surface was analyzed using scanning tunneling microscopy (STM). To obtain a sufficiently large database for the relatively rare and rapid events reported here, typically $10-15 \mathrm{~h}$ of permanent STM recording is required. This task calls for a special STM setup with highest thermal stability and a base pressure in the vacuum chamber of below $5 \times$ $10^{-11}$ mbar. For this purpose, we have developed a new STM based on the Besocke design [20] with a ceramic baseplate, which allows for high precision measurements at variable temperatures for more than $20 \mathrm{~h}$ on essentially the same surface area. The island sizes are analyzed using special computer codes. The programs, as well as the details of the sample preparation, are described elsewhere [13]. After preparation, the mean terrace width of the surface was $0.5-1 \mu \mathrm{m}$ and the density of pinning sites was $10^{-7}$ per area of an atom. The surface remained clean even after $10-15 \mathrm{~h}$ of observation.

Several monolayers of copper were deposited using a self-calibrating evaporator (Omicron EFM 3) while the surface was held at $314 \mathrm{~K}$. During deposition, the pressure never exceeded $2 \times 10^{-10}$ mbar. Under these conditions, mounds of 2-4 monolayers height are formed. The decay was observed in more than 800 STM images $\left(4000 \times 4000 \AA^{2}\right)$ covering a time span of $13 \mathrm{~h}$ while the temperature was kept at $314 \mathrm{~K}$. The scan time for each 
$512 \times 512$ pixel image was about 1 min. The STM images in Fig. 1(a) focus on a particular double layer island close to a step edge. The time span between the first and the last image shown in Fig. 1 is about $4.5 \mathrm{~h}$. The initial areas of the top and the second layer islands correspond to about 6000 and 13000 atoms, respectively.

In image I of Fig. 1(a), the top layer island is approximately in the center of the second layer island. Both islands decay in time. The decay rate of the second layer island increases as the island size becomes smaller following a $\left(t-t_{0}\right)^{\alpha}$ power law with $\alpha \cong 0.55$, which is typical for the diffusion limited decay of monolayer high islands on $\mathrm{Cu}(111)$ in the presence of other larger islands on terraces $[13,18]$. The mean decay rate up to $t=4.5 \times 10^{4} \mathrm{~s}\left[\nu=-0.12 \mathrm{~s}^{-1}\right.$, Fig. 1(b) $]$ is consistent with the results in [13]. The decay of the top layer

(a)
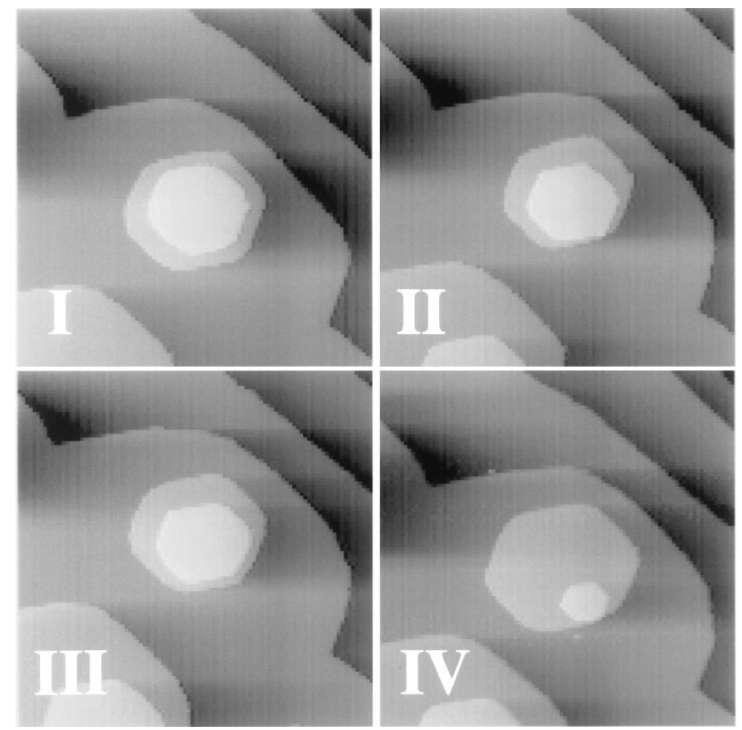

(b)

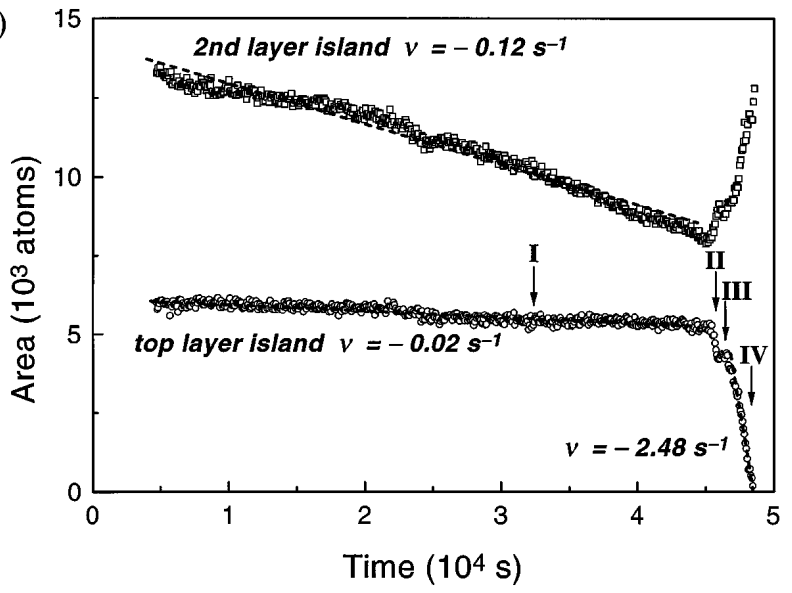

FIG. 1. (a) STM images at $T=314 \mathrm{~K}$ of a double layer island at different times indicated in (b). The images show a section of $800 \times 800 \AA^{2}$ of the original $4000 \times 4000 \AA^{2}$ images, each displaying about 50 multilayer islands. (b) Area of both layers of the double island, shown in (a), plotted vs time. The dashed lines are linear fits to determine the decay rates $\nu$. island $\left(\nu=-0.02 \mathrm{~s}^{-1}\right)$ is slower since its decay involves hopping over the step edge barrier. The same slow decay was also found in this work for several other isolated islands of the same size placed on top of islands.

Around $300 \mathrm{~K}$, even large islands, like the top layer island in Fig. 1(a), engage in a random walk via rapid edge diffusion which has been shown to be the predominant mass transport on $\mathrm{Cu}(111)$ up to $500 \mathrm{~K}$ [21]. Because of the random walk, the top layer island touches the second layer island edge as shown in image II of Fig. 1(a). At this particular point in time, marked as II in Fig. 1(b), the decay rate is enhanced by 2 orders of magnitude $(\nu=$ $-2.48 \mathrm{~s}^{-1}$ ). The rapid decay continues as long as the top layer island sticks to the edge of the island below. When the ledge contact breaks, the decay rate becomes lower (III) until the island touches the edge again (IV). The rapid decay events were observed whenever the STM image indicated a ledge contact. In total, 20 events were analyzed so far, including several ledge contacts for vacancy islands in vacancy islands and about the same decay rate was found in all cases.

The formation of a ledge contact and the reengagement in a random walk are stochastic processes which are highlighted in Fig. 2. The initial island configuration consists of two islands of comparable size (located within the white circle) which are located on top of a large island [image I, Fig. 2(a)]. A ledge contact exists between all three layers and, consequently, the decay of the top layer island and the second layer island is fast [Fig. 2(b)]. Their decay rates are identical within the limits of error $(\nu=$ $-1.42 \mathrm{~s}^{-1}$ ). Later in time, the ledge contact is broken [image II of Fig. 2(a)] and the decay rates of both islands are low [marked as II in Fig. 2(b)]. At about $t=3.9 \times$ $10^{4} \mathrm{~s}$, the top layer island touches the edge of the second layer island [image III of Fig. 2(a)]. Simultaneously, the decay rate of the top layer island increases by 2 orders of magnitude to $\nu=-2.41 \mathrm{~s}^{-1}$. In image IV of Fig. 2(a), the ledge contact is broken again and the top layer island has engaged in a random walk. Then, as well as in the time range around $2 \times 10^{4} \mathrm{~s}$ (II), the rate is as for normal diffusion limited decay for islands on islands. The oscillations in the size of the second layer island after the initial rapid decay reflect the influence of the other islands on the third layer [see image II of Fig. 2(a)] as well as the decay of the top layer island.

So far, we have considered ledge contacts which occur stochastically because of the random walk of the top layer island. In addition, ledge contacts may be forced by the shrinking size of a lower layer island. An example for this scenario is shown in Fig. 3 with the decay of a triple layer island on a narrow terrace (inset of Fig. 3). Up to about $10^{4} \mathrm{~s}$, the top layer and the second layer island decay with reduced rates because of the SE barrier, while the third layer island decays much faster since it loses atoms to the ascending step nearby in the standard diffusion limited decay. At about $10^{4} \mathrm{~s}(\mathrm{I})$, the third layer island approaches the size of the second layer island and the decay of the 
(a)

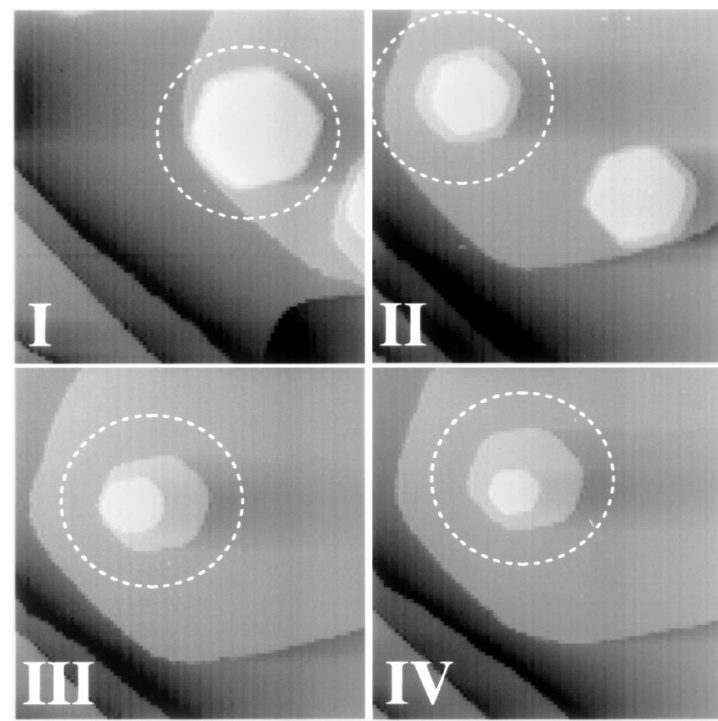

(b)

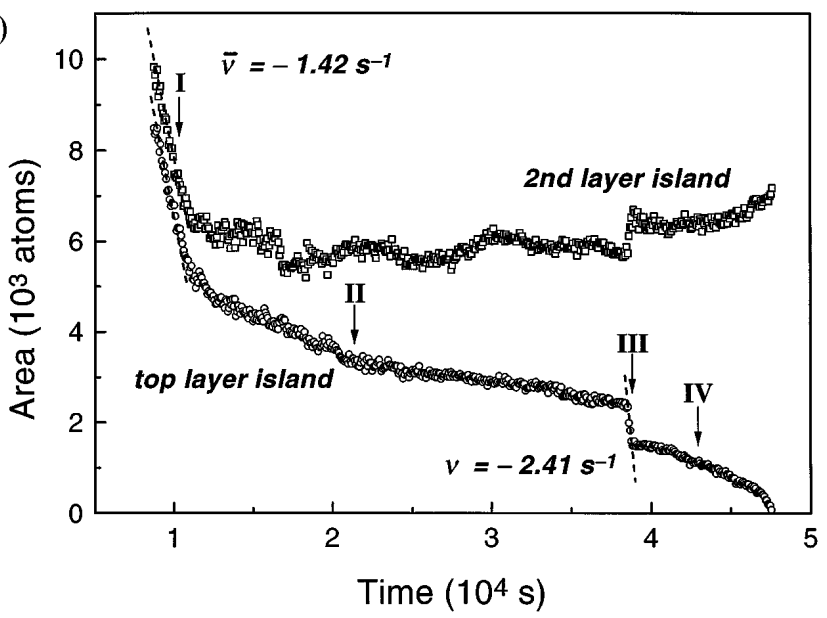

FIG. 2. (a) STM images of a double layer island (white circle). The images show a section of $730 \times 730 \AA^{2}$ of the same data as in Fig. 1. (b) Area of both layers of the double island, shown in (a), plotted vs time. The dashed lines are linear fits to determine the decay rates $\nu$.

second layer island is accelerated. At around $2 \times 10^{4} \mathrm{~s}$ (II), the second and the third layer islands have about the same size as the top layer island. Then, the decay of the top layer island is also accelerated and all three islands decrease in size with about the same rate. The decay rate for the top layer islands is smaller than decay rates for the ledge contact decay in Figs. 1 and 2. This lower rate results from the rather small difference in the radii of the islands in the case of forced ledge contacts: Whenever the fluctuations cause a local step contact, atoms flow from the upper island to the lower. Thereby, the local curvature of the upper islands is reduced, while the local curvature of the lower island increases. The initially small difference in the chemical potential between the upper and the lower island, therefore, vanishes after only a small mass transfer to the lower islands. This leads to a smaller average decay rate compared to the case where the top island is significantly smaller. The decay of the lowest layer island
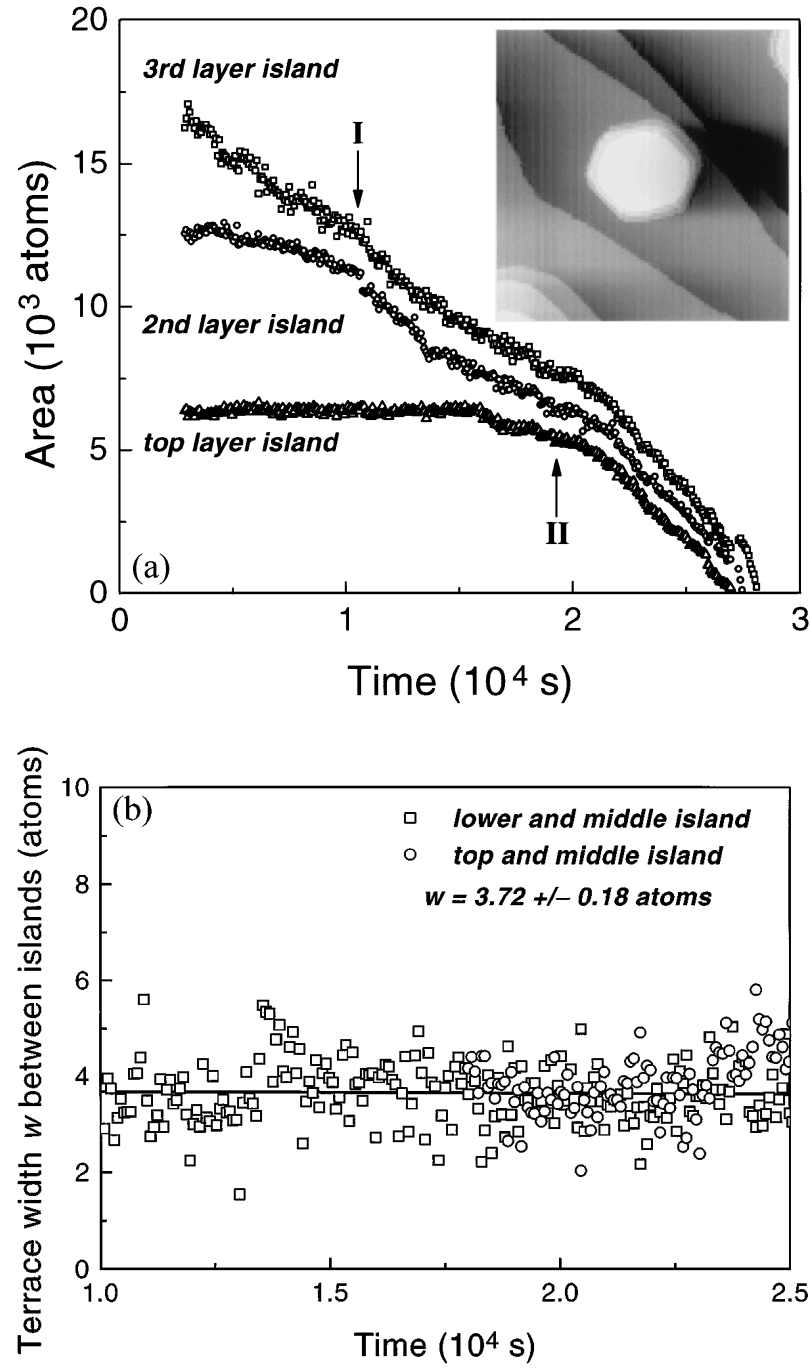

FIG. 3. (a) Area decay of a triple layer height mound. The inset shows a $780 \times 780 \AA^{2}$ section of an STM image at $t=1.4 \times 10^{4} \mathrm{~s}$. (b) Terrace width between the islands in (a) when the decay is step contact controlled.

results from the balance between the diffusion limited decay to the neighboring step and the gain from the islands above. The decay of the lowest island is, therefore, slower than the normal diffusion limited decay which obeys a $\left(t-t_{0}\right)^{0.55}$ law [13]. During the final stages of the decay, the width of the terraces between the ledges of the islands remains constant at a mean width of 3.72 atom diameters [Fig. 3(b)]. The scattering of the data is mostly due to error in the determination of the island areas in the triple layer island. The terrace width is presumably controlled by the magnitude of the step fluctuations $[17,21]$ and the decay rate of the lowest island in combination with the step contact decay mechanism.

In order to elucidate the reason for the sudden increase of the decay rate, we have performed computer simulations of the diffusion limited decay for an island placed on top of another island [22]. In particular, we have investigated the decay as a function of the position of the top island on the island below. We found that, in the 
presence of a high SE barrier, the decay rate of the top island is rather insensitive to its position. In particular, there is hardly an increase when the top island approaches the step edge of the lower island. This result is consistent with the constant decay rate of the top layer island in Fig. 1(b) during the time before ledge contact. When the SE barrier is set to zero in the simulations, the decay depends critically on the position and increases proportionally to the inverse of the distance when the island approaches the lower island edge. Hence, the simulations predict a large increase of the decay rate when the distance between the island edges becomes small and the SE barrier is removed. The experimental results can, therefore, qualitatively be interpreted by an absence of the SE barrier for islands with step edges in close proximity. Quantitative information cannot be extracted from these simulations, since the algorithm is based on a continuum approximation which is not valid for small distances between island edges. Unfortunately, the microscopic structure of a ledge contact could not be determined. Establishing an island decay as ledge contact is controlled requires the quantitative analysis of large scale images which are not atomically resolved. The microscopic structure of ledge contacts could be that of a low index microfacet. Then, the decay from the upper to the lower layer island may involve an exchange process of atoms not hindered by an SE barrier. Another possible scenario is that of a ledge contact consisting of two steps at close distance. Then, the overlapping local strain fields originating from the steps could remove the SE barrier.

The ledge contact decay channel has significant consequences for the stability of mounds. A normal diffusion limited decay of mounds would proceed via a sequential decay of the islands from the top to the bottom, the decay of each layer being slowed down by the SE barrier. Because of the ledge contact mechanism, the entire mound disappears with the decay rate of the lowest layer island which is not slowed down by an SE barrier. For mounds consisting of a large number of layers, the time for a total decay is, therefore, reduced by orders of magnitude. This rapid decay should have significant consequences for the long time scaling behavior in epitaxial growth. For mounds engaged in the ledge contact decay mode, the slope should be controlled by the edge fluctuations of the islands. Since fluctuations increase with temperature, the slope should become less steep at higher temperatures. This opens interesting new prospects for the preparation of mounds with a controlled morphology.
The authors acknowledge the skillful crystal preparation by U. Linke.

*Corresponding author.

Electronic address: m.giesen@fz-juelich.de

[1] G. Ehrlich and F. G. Hudda, J. Chem. Phys. 44, 1039 (1966).

[2] R. L. Schwoebel and E. J. Shipsey, J. Appl. Phys. 37, 3682 (1966).

[3] J. E. Van Nostrand, S. J. Chey, M. A. Hasan, D. G. Cahill, and J. E. Greene, Phys. Rev. Lett. 74, 1127 (1995).

[4] M. Siegert and M. Plischke, Phys. Rev. Lett. 73, 1517 (1994); P. Smilauer and D. D. Vvedenski, Phys. Rev. B 52, 14263 (1995).

[5] J. G. Amar and F. Family, Phys. Rev. B 54, 14742 (1996); M. C. Bartelt and J. W. Evans, Phys. Rev. Lett. 75, 4250 (1995).

[6] G. S. Bales and A. Zangwill, Phys. Rev. B 41, 5500 (1990).

[7] H. J. Ernst, F. Fabre, R. Folkerts, and J. Lapujoulade, Phys. Rev. Lett. 72, 112 (1994); L. Schwenger, R. L. Folkerts, and H. J. Ernst, Phys. Rev. B 55, R7406 (1997).

[8] R. Kunkel, B. Poelsema, L. K. Verheij, and G. Comsa, Phys. Rev. Lett. 65, 733 (1990).

[9] F. Tsui, J. Wellman, C. Uher, and R. Clarke, Phys. Rev. Lett. 76, 3164 (1996).

[10] H. A. van der Vegt et al., Phys. Rev. Lett. 68, 3335 (1992).

[11] W. Wulfhekel et al., Surf. Sci. 348, 227 (1996).

[12] K. Morgenstern, G. Rosenfeld, E. Lægsgaard, F. Besenbacher, and G. Comsa (to be published).

[13] G. Schulze Icking-Konert, M. Giesen, and H. Ibach, Surf. Sci. (to be published).

[14] R. Stumpf and M. Scheffler, Phys. Rev. Lett. 72, 254 (1994).

[15] Yinggang Li and A.E. DePristo, Surf. Sci. 319, 141 (1994).

[16] J. B. Hannon, C. Klünker, M. Giesen, H. Ibach, N. C. Bartelt, and J.C. Hamilton, Phys. Rev. Lett. 79, 2506 (1997).

[17] M. Giesen-Seibert, F. Schmitz, R. Jentjens, and H. Ibach, Surf. Sci. 329, 47 (1995).

[18] J. M. Wen, S.-L. Chang, J. W. Burnett, J. W. Evans, and P. A. Thiel, Phys. Rev. Lett. 73, 2591 (1994); K. Morgenstern, G. Rosenfeld, B. Poelsema, and G. Comsa, Phys. Rev. Lett. 74, 2058 (1995).

[19] S. Khare and T.L. Einstein, Phys. Rev. B 54, 11752 (1996).

[20] K. H. Besocke, Surf. Sci. 181, 145 (1987).

[21] M. Giesen, G. Schulze Icking-Konert, and H. Ibach (to be published).

[22] For technical details, see Ref. [13]. 